Anuario Latinoamericano Ciencias Políticas

y Relaciones Internacionales

vol. 9, 2020

pp. 173-193

\section{Elecciones presidenciales en El Salvador 2019: la derrota del FMLN y un nuevo gobierno con Nayib Bukele}

\author{
Presidential Elections in El Salvador 2019. \\ The Defeat of the FMLN and a New Government \\ with Nayib Bukele
}

\author{
Ignacio Medina Núñez \\ EL COLEGIO DE JALISCO \\ ZAPOPAN, MÉXICO \\ $\triangle$ nacho@coljal.edu.mx
}

http://orcid.org/0000-0002-1660-2327

\title{
RESUMEN
}

Cada cinco años se realizan elecciones presidenciales en El Salvador, en Centroamérica. Cada tres años se realizan elecciones para diputados y presidentes municipales. Dos períodos consecutivos de gobernar el Frente Farabundo Martí para la Liberación Nacional (FMLN) en el país (2009 a 2019) mostraron que los líderes de la antigua organización guerrillera llegados al gobierno fracasaron en su propuesta de desarrollo económico y político. Las elecciones legislativas de 2018 mostraron entonces que podrían perder la presidencia para las elecciones nacionales del año siguiente. Efectivamente, surgió una novedosa opción política para el país con la elección del nuevo presidente electo Nayib Bukele en 2019. Después del terrible conflicto bélico de la década de 1970 y 1980 en El Salvador, llegó a gobernar el país durante 20 años, en 4 períodos presidenciales, la opción de ultraderecha de la Alianza Republicana Nacionalista (ARENA), pero la antigua organización guerrillera, el FMLN, recibió el apoyo de la población para gobernar a partir del 2009. Con un método de análisis documental (libros, artículos, encuestas y estadísticas) y a partir de entrevistas con investigadores del área centroamericana, nos preguntábamos el por qué la opción

\footnotetext{
* Doctor en Ciencias Sociales por la Universidad de Guadalajara, México. Profesor investigador en El Colegio de Jalisco, México, y coordinador del Doctorado en Ciencias Sociales. Miembro del Sistema Nacional de Investigadores del Consejo Nacional de Ciencia y Tecnología (CONACYT) desde 1990. Estuvo un año como profesor invitado en la Universidad Estatal de Georgia, Atlanta, GA, en Estados Unidos, con la beca Fullbright-García Robles. En 2012, recibió la Presea al Mérito Académico "Enrique Díaz de León” por el Sindicato de Trabajadores Académicos de la Universidad de Guadalajara (STAUDEG).
} 
del FMLN, que había sido una organización guerrillera y que pudo llegar a tener la presidencia del país, provocó un desencanto en la población después de dos períodos consecutivos de gobierno. La respuesta la obtuvimos en la insatisfacción generalizada por la creciente violencia en el país, por las divisiones internas de la organización y por una política social que no pudo reflejarse en la mejoría en la calidad de vida de los salvadoreños. Nuestra conclusión apunta a la gran dificultad de gobernar de un gobierno de izquierda que, en este caso, no pudo mantener sus promesas fundamentales y cómo los ciudadanos pueden ejercer un voto de castigo y optar por nuevas opciones. Se mantiene, además, la pregunta sobre el nuevo proyecto de país que representa la figura emergente del nuevo presidente electo.

PALABRAS CLAVE: El Salvador, elecciones, democracia, desarrollo.

\section{ABSTRACT}

Every five years presidential elections are held in El Salvador, in the Central American region. Elections for deputies and municipal presidents are held every three years. Two consecutive periods of the government of the Farabundo Martí National Liberation Front (FMLN) in the country (2009 to 2019) showed that the leaders of this former guerrilla organization failed in their proposal for economic and political development. The 2018 legislative elections then showed that they could lose the presidency for the national elections of the following year. And, indeed, a new political option emerged for the country with the election of the new president Nayib Bukele in 2019. After the terrible war conflict of 1970 s and 1980s, the ultra-right Republican Nationalist Alliance (ARENA) governed El Salvador for 20 years; but finally, the FMLN conquered the support of the people in 2009. Using a method of documentary analysis (books, articles, surveys and statistics) and interviewing researchers on the Central American area, we tried to find out why the FMLN option, which had been a guerrilla organization and could have had the presidency of the country, caused a disenchantment in the population after two consecutive periods of government. We got the answer in the general dissatisfaction with the growing violence in the country, the internal divisions

of the organization and a social policy that did not lead to an improvement of life of Salvadorans. Our conclusion points to the great difficulty a leftist government had in keeping its fundamental promises and how citizens can exercise a punishment vote and opt for new options. In addition, the question remains about the new project for the country that the emerging figure of the new president-elect Nayib Bukele represents.

KEYWORDS: El Salvador, elections, democracy, development.

\section{Introducción}

La situación social y política contemporánea de El Salvador debe ser considerada a partir del contexto de la guerra entre el gobierno salvadoreño y la organización político-militar insurgente del Frente Farabundo Martí para la 
Liberación Nacional (FMLN) que se llevó a cabo en el país en las décadas de 1960, 1970 y 1980, hasta llegar a los Acuerdos de Paz de 1992. Fue un proceso sangriento de violencia que dejó 75.000 mil víctimas en 12 años de conflicto bélico ${ }^{1}$.

Con el resultado de los acuerdos de Paz de 1992, después de la confrontación bélica, se terminó formalmente la guerra y se impulsaron reformas institucionales para permitir la inclusión de los combatientes, una mayor libertad de expresión, un marco de mejor respeto a los derechos humanos y un mejor desarrollo económico. De haber sido una organización guerrillera, el FMLN se transformó en partido político y pudo, en la última década del siglo XX, participar ya en las contiendas político-electorales.

Se realizaron las primeras elecciones presidenciales en el nuevo contexto de paz en 1994. Las ganó la Alianza Republicana Nacionalista (ARENA), partido que volvió a triunfar en 1999 y en el 2004. Sin embargo, en 2009, por primera vez ganó la presidencia el FMLN con un candidato externo, el periodista y presentador de televisión, Mauricio Funes, y por segunda vez, en 2014, con un candidato propio, Salvador Sánchez Cerén, quien en el período de la guerra había sido combatiente con el nombre de Comandante Leonel.

Este artículo pretende ofrecer un contexto político sobre los dos gobiernos del FMLN en El Salvador para tratar de explicar por qué perdió la presidencia y qué significado tiene la opción ganadora para el período 2019-2024. Se trata de responder a la principal interrogante: ¿Por qué fracasó electoralmente la opción surgida del movimiento guerrillero del FMLN ante la propuesta de un candidato que fue expulsado de sus propias filas, pero aliado con sectores de la derecha política?

Desde la antigua Grecia, el concepto de democracia, o República (como la llamaron los romanos) hasta la época moderna -con excepción de todo el período del feudalismo- ha tenido un sentido inspirador en cuanto a la participación del pueblo en los asuntos públicos, con el objeto de no dejar la dirección de una nación en manos de un solo dirigente. Especialmente desde el siglo XVII, cuando Spinoza (2015) recuperó el modelo de la democracia como el mejor comparado con la monarquía y la aristocracia, hasta la época contemporánea cuando Alain Touraine (2001) la define -junto con la realización de

1 Después de la firma de los Acuerdos de Paz en 1992, se creó la Comisión de la Verdad con el fin de investigar los hechos violentos ocurridos en el país. En esta Comisión se integraron Belisario Betancourt, Reinaldo Figuereido, Thomas Buergenthal, y otras personalidades. Se presentó el informe final en la ONU en marzo de 1993, titulado De la locura a la esperanza: la guerra de doce años en El Salvador (ONU, 1993). Ahí se encontró que el 95 \% de los casos de desaparecidos y muertos en el país en el transcurso de la guerra fueron causados por el gobierno de El Salvador, el Ejército y los llamados escuadrones de la muerte, mientras que el FMLN también fue causante del $5 \%$ de los casos violentos. Aunque hubo masacres en pueblos enteros, llamó mucho la atención internacional el asesinato de Mons. Óscar A. Romero, el de cuatro religiosas norteamericanas (tres monjas y una misionera laica) en 1980 y el de los Jesuitas de la Universidad Centroamericana en 1989.
Elecciones presidenciales en EI Salvador 2019: la derrota del FMLN y un nuevo gobierno con Nayib Bukele

Ignacio Medina Núñez 
elecciones reales- como un régimen donde "una mayor cantidad de personas participa directa o indirectamente en la toma de decisiones" (Touraine, 2001, p. 3), encontramos una multitud de elementos para lograr una concepción que va más allá de los procesos electorales. Es por ello que, al hablar de la calidad de las democracias latinoamericanas, Morlino encuentra a "El Salvador, Guatemala, Paraguay, Nicaragua y Venezuela" en una clasificación de "democracias de inferior calidad" (Morlino, 2014, p. 60). La misma ONU, cuando múltiples países han llegado a la elección de los gobernantes a través del voto de los ciudadanos, enfatiza la necesidad, más allá de las elecciones, de profundizar este modelo en un mundo fragmentado: "Para que las políticas y las instituciones políticas promuevan el desarrollo humano y protejan la libertad y la dignidad de todas las personas, se ha de ampliar y consolidar la democracia" (PNUD, 2002, p. 1). Sin embargo, el hecho mismo de que los ciudadanos puedan ejercer el voto ya significa un gran avance para enfrentar toda una situación de dictaduras que caracterizaron América Latina por varias décadas.

En este momento no profundizaremos en los grandes significados teóricos del modelo democrático ${ }^{2}$, sino que nos ocuparemos sólo de la importancia del voto ciudadano en el momento determinado de un país porque los procesos electorales siguen siendo un tema de vital importancia para la democracia y para la ciencia política. El caso político electoral de El Salvador en 2019 refleja una situación generalizada en muchos países cuando los partidos tradicionales de derecha e izquierda se han visto desplazados, y han surgido movimientos emergentes. La inconformidad electoral de la población se manifiesta en nuevas opciones políticas cuyo proyecto puede ser novedoso con tendencias variadas en el panorama latinoamericano. Por ello, aunque es un país pequeño en el área centroamericana, El Salvador cobra una enorme importancia en la discusión sobre el devenir del continente con disputas político-electorales y nuevas propuestas de cambio social.

\section{Gobierno del FMLN con Mauricio Funes (2009-2014)}

El triunfo del FMLN, después de 20 años de gobiernos de la derecha con ARENA, al alcanzar por primera vez la presidencia del país en 2009 , fue novedoso en un contexto latinoamericano donde habían ocurrido triunfos de fuerzas de izquierda en otros países (Medina, 2009, pp. 123-270). El fracaso de las promesas del gobernante anterior de ARENA, Antonio Saca, fue evidente: crecía la violencia y la delincuencia en el país, la economía no despegaba más

2 Ya en otros momentos nos hemos ocupado de profundizar teóricamente el concepto de democracia, empezando por la Grecia antigua (Medina, 2005) hasta el surgimiento del siglo XXI (Medina, 2018). Aquí solamente trataremos de enfatizar la importancia de la democracia electoral, porque ahí se determina el rumbo en los períodos que tiene el gobierno de cada país, que en el caso de El Salvador es un tiempo de cinco años. 
allá de un crecimiento del $2 \%$. Una minoría seguía enriqueciéndose mientras que en la mayoría de la población había penurias, violencia, aumentaba el desempleo y también la migración con rumbo a México y Estados Unidos. La represión violenta con golpes, gas y balas de goma sobre las manifestaciones públicas en las calles fue otro signo del gobierno de Saca.

En 2009, se enfrentaron los candidatos de los dos partidos más fuertes: con acierto, el FMLN había postulado a Mauricio Funes, hombre progresista que, sin ser militante de ese partido, había aceptado la candidatura; con desacierto, ARENA había postulado a Rodrigo Ávila, que tenía de antemano una imagen negativa por su ineficacia y su actuación represiva anterior al frente de la Policía Nacional Civil. El gobierno de Saca, el partido ARENA con el apoyo e intervención de la Embajada de Estados Unidos centraron su campaña en el miedo al socialismo y al comunismo, y lograron atraer al PDC y al PCN para hacer una alianza electoral. Pero fue la figura civil de Mauricio Funes y su trabajo público con una visión progresista en la televisión la que fue ganando la aceptación de los salvadoreños, decepcionados ya de cuatro gobiernos consecutivos de ARENA desde 1989.

El FMLN triunfó en 2009, pero no con la contundencia que se había predicho en las encuestas. No hubo necesidad de una segunda vuelta electoral puesto que el 15 de marzo Funes consiguió el 51,32 \% de los votos, mientras que ARENA el 48,68 \% para Rodrigo Ávila, habiendo solamente 69.412 votos de diferencia (PDA, 2009).

Históricamente el ascenso electoral del FMLN era notable después de la firma de los Acuerdos de Paz: Rubén Zamora el $24 \%$ en la primera vuelta, Facundo Guardado el 29 \%, Schafick Handal el 35 \%, y finalmente Mauricio Funes el $51 \%$. Sin embargo, en el 2009, el poder total era compartido: el FMLN había logrado 35 diputados mientras que ARENA 32; el PCN y el PDC, aliados de ARENA, conquistaron 11 y 5 respectivamente. De esta manera, ARENA con la fuerza de sus pequeños aliados tenía 48 diputaciones frente a 35 del FMLN. Y, además, aunque el partido ganador de la presidencia había conquistado mayor número de presidencias municipales, perdió la más significativa, la capital San Salvador. La tarea se veía clara desde un principio, sobre todo en cuanto a lo que no debería de hacer el gobierno, como lo mencionó Funes en su toma de posesión: "no hacer lo que ya algunos hicieron mal en este país: gobernar para pocos, ser complaciente con la corrupción, temer y ser cómplice del crimen organizado, pactar con el atraso en todas sus formas de expresión" (Funes, 2009).

En junio de 2009, el presidente Funes tenía una popularidad del $80 \%$ y en un contexto de gran esperanza de la población sobre gran cambio para mejorar. El énfasis en los aspectos sociales como la salud, la educación y la lucha contra la pobreza se dejaron sentir desde un inicio, pero el modelo macroeconómico imperante siguió vigente: "En el aspecto económico, el gabinete de Funes ha optado por alinearse ante los organismos multilaterales como el Banco Interamericano de Desarrollo (BID), el Fondo Monetario Internacional
Elecciones presidenciales en EI Salvador 2019: la derrota del FMLN y un nuevo gobierno con Nayib Bukele Ignacio Medina Núñez 
(FMI) o el Banco Mundial (BM). Esto ha transmitido un sentimiento de alivio a la Asociación Nacional de la Empresa Privada (ANEP), así como a la dirigencia del mayor partido opositor de derecha, ARENA, y a los también derechistas Partido de Conciliación Nacional (PCN) y Partido Demócrata Cristiano (PDC)" (La Universidad, 2009, p. 2). Así, el gobierno del FMLN, presidido por Mauricio Funes, enfatizó los aspectos sociales, pero no hubo en realidad un nuevo proyecto de país mientras que dos problemas seguían perturbando a los salvadoreños: por un lado, la delincuencia e inseguridad y, por el otro, la corrupción e impunidad.

En mayo de 2010, a casi un año del gobierno de Funes, el Instituto Universitario de Opinión Pública (IUDOP, 2010) de la Universidad Centroamericana Simeón Cañas en San Salvador realizó una encuesta sobre el presidente, sobre el aparato legislativo y las alcaldías. Algunas de sus conclusiones fueron las siguientes: el principal problema de El Salvador era la delincuencia (45,3\%), y seguía la economía (17,8 \%) y la violencia (10,7 \%). El 58,3 \% de los encuestados señalaba que había una mejoría en el país mientras que el 41,7 \% no veía nada positivo. El principal logro de Funes era la educación (40 \%) mientras que el 27,6 \% no veía ninguno. El principal fracaso del presidente lo veían en el combate a la delincuencia (26,7\%). Además, para el 48,8 \% el país seguía igual, para el 24,2 \% había mejorado, y para el 25,5\% la situación estaba peor que antes.

El mismo IUDOP (2014b), con la misma metodología, realizó otra encuesta del 3 al 9 de mayo de 2014, posterior al nuevo triunfo del FMLN en la presidencia del país. La nota general de evaluación era de 6,59 puntos porque el 53,1 \% tenía una imagen positiva del presidente Funes, y 61,2 \% pensaba que había gobernado bien, y además el 61,9\% creía que se había realizado un cambio positivo en el país. En cuanto al gran logro del gobierno, el 38,5 \% de los encuestados lo ubicaba en el área de la educación, pero sólo el 1,5 \% pensaba que algo se había mejorado en el ámbito de la delincuencia, mientras que el 35,5\% creía que era uno de sus mayores fracasos, porque el 71,3 \% veía que el problema había aumentado. Sólo el 26,5 \% estaba convencido de que había cumplido con sus promesas de campaña, mientras que el $29,8 \%$ señalaba que no había cumplido.

Así, el FMLN en el gobierno de Funes fue aprobado con una calificación mínima que logró pasar la dirección del gobierno al mismo partido con el candidato Salvador Sánchez Cerén en las elecciones presidenciales de 2014. Todo mundo podía reconocer mejoras en el ámbito educativo, pero ni la economía había mejorado ni la delincuencia había disminuido ni había ocurrido una disminución real en el número de pobres en el país. En el ámbito de la delincuencia, había sucedido una estrategia del gobierno muy controvertida porque se había propiciado una tregua entre pandillas desde marzo del 2012 con negociaciones directas con los grupos delictivos, transfiriéndoles incluso recursos económicos con la intención de evitar grados extremos de violencia. 


\section{Gobierno del FMLN con Salvador Sánchez Cerén: 2014-2019}

El FMLN ganó la presidencia del país por segunda vez en 2014 pero de una forma más compleja y difícil con un candidato propio: Salvador Sánchez Cerén había sido combatiente con el nombre de Comandante Leonel en el tiempo de la guerra contra el gobierno, dirigiendo a las Fuerzas Populares de Liberación (FPL) y luego dentro del FMLN en lucha contra el gobierno de Napoleón Duarte (PDC) y de Alfredo Cristiani (ARENA).

Las elecciones se realizaron el 2 de febrero de 2014, y ganó el FMLN con $48,92 \%$ de los votos, mientras que ARENA con su candidato Norman Quijano consiguió el 38,95 \%. La coalición Unidad (GANA, PDC, PCN), donde participó el partido que ganaría luego la presidencia en el 2019, conquistó el 11,44 \% con el expresidente Saca; el PSP ${ }^{3}$ el 0,44 \%, y el FPS el 0,26 \%. El FMLN ganó 13 de los 14 departamentos. El proceso estuvo marcado por un abstencionismo de más del $50 \%$ en la primera vuelta.

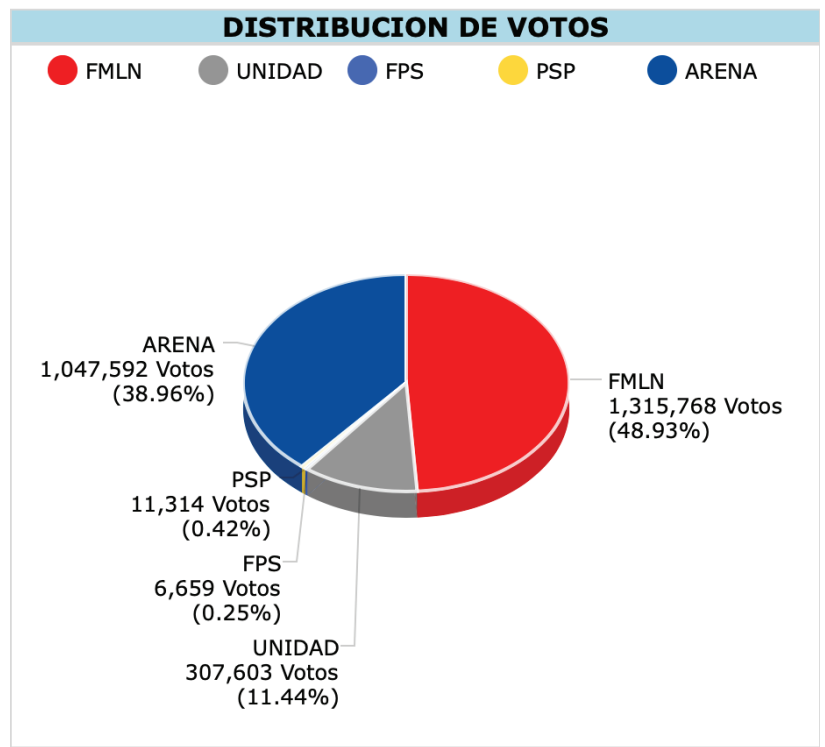

Fuente: Escrutinio final del Tribunal Supremo Electoral (TSE, 2014). https://www.tse.gob. sv/2014/escrutiniofinal_1ray2davuelta/index.html

\footnotetext{
3 El Partido Salvadoreño Progresista (PSP) se califica a sí mismo en una posición de centro progresista. Para el 2015 se alió con el FMLN para ganar la alcaldía de San Salvador con Nayib Bukele como candidato (aunque con sus propias siglas solamente aportó 3.375 votos). En el 2018, el TSE le canceló el registro legal.

4 El partido Fraternidad Patriota Salvadoreña (FPS) fue una organización de derecha que se fundó en 2011, pero al que el TSE le quitó el registro legal en 2018.
}

Elecciones presidenciales en El Salvador 2019: la derrota del FMLN y un nuevo gobierno con Nayib Bukele Ignacio Medina Núñez

Gráfico 1.

Elecciones presidenciales 2014 en El Salvador 
Artículos y ensayos

Gráfico 2.

Elecciones presidenciales 2014 en El Salvador (la segunda vuelta)
De acuerdo con la legislación electoral, al no obtener ningún candidato más del $50 \%$ de la votación, se tuvo que programar una segunda vuelta para el 9 de marzo de 2014 sólo entre los dos candidatos que lograron la mayor votación (FMLN y ARENA); ambos querían atraer a la importante tercera fuerza de Unidad y sus poco más de 300 mil votos. Realizada la segunda vuelta, el escrutinio final fue muy apretado pero el TSE otorgó al FMLN con el 50,11 \% (1.495.815 votos) de la votación, mientras que ARENA obtuvo el 49,89 \% (1.489.451 votos). La diferencia final fue de 6.364 votos. De una población total de 6,3 millones de habitantes fueron 4,9 millones convocados a votar, restando 650.181 que no renovaron su Documento Único de Identidad. En la segunda vuelta acudió el $60 \%$ de los votantes, pero con una votación tan cerrada que no hubo resultados oficiales sino hasta el domingo 16 de marzo con el escrutinio y decisión final del TSE. El triunfo del FMLN, por tanto, en una segunda ocasión, después del primer período de gobierno con Mauricio Funes, no fue contundente como lo había sido en 2009. Este hecho, por sí mismo, debía de haber advertido al partido gobernante que algo no había funcionado bien en la etapa anterior. Sin embargo, como veremos, la tendencia negativa, más que corregirse, se profundizó con el ejercicio de Sánchez Cerén en el poder ejecutivo del país.

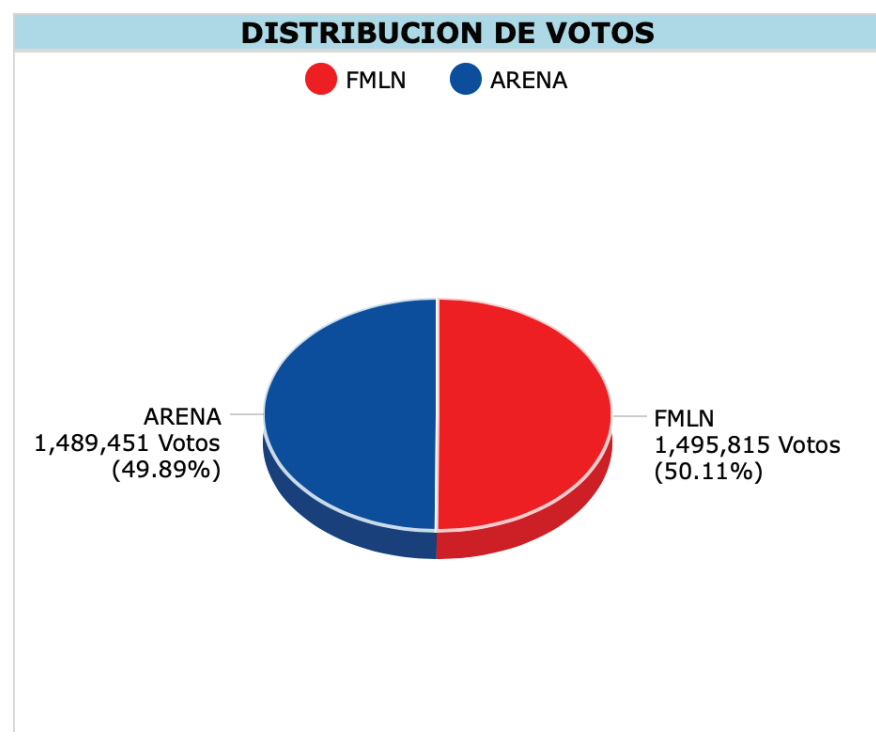

Fuente: Escrutinio final del Tribunal Supremo Electoral (TSE, 2014). https://www.tse.gob. sv/2014/escrutiniofinal_1ray2davuelta/index

En este proceso electoral presidencial ya participó como partido Gran Alianza por la Unidad Nacional (GANA), una organización que como grupo se había escindido de ARENA después que este partido sufrió una cri- 
sis al perder la elección presidencial del $2009^{5}$ frente al FMLN. El grupo de GANA quiso separarse de la tendencia ultraderechista del exmilitar Roberto d'Aubuisson, pero mantuvieron una ideología conservadora y de derecha y solamente querían mantenerse lejos de los actos de corrupción de los gobernantes de ARENA ${ }^{6}$. Para las elecciones presidenciales del 2014, GANA se había aliado con el PDC y el PCN (también partidos conservadores) para postular al expresidente Elías Antonio Saca, pero, aunque lograron 305.295 votos, no lograron pasar a la segunda vuelta de marzo 2014.

$\mathrm{Al}$ acercarse la toma de posesión de Sánchez Cerén como presidente en junio 2014, la mayoría de los salvadoreños seguía creyendo que la principal tarea gubernamental debía ser el combate a la delincuencia, la creación de fuentes de empleo y mejorar la economía nacional. El 80,1 \% de los encuestados insistía en que había que cambiar la estrategia de seguridad pública, y el $70 \%$ en que debería de cambiar la política económica. De cualquier forma, el 50,5 \% tenía la esperanza de que la situación del país iba a mejorar con el segundo triunfo del FMLN, y el 53,1 \% confiaba mucho en la persona del presidente electo (IUDOP, 2014b).

Para el 2016, la economía no mejoraba7: no había transparencia en la administración pública y los beneficios sociales no eran significativos (Arauz, Peña y Burgos, 2016). Y el problema más impactante seguía siendo la violencia y la delincuencia porque en 2015, con los indicadores de la ONU, el país se había convertido en el país más violento de América Latina. El indicador internacional reflejado en la cifra de homicidios por cada 100 mil habitantes es una importante referencia para medir el problema: cuando se rebasa más de 20 el número de homicidios, se puede hablar de un problema gravísimo. Con los gobiernos de ARENA la cifra de asesinados en el año 2000 fue de 45,5 asesinados por cada 100 mil, y el número se elevó hasta 51,9 en el año 2008.

Mauricio Funes estableció una estrategia diferente con negociaciones, pactos y tregua con las pandillas, lo cual funcionó relativamente bien cuando vemos que no empeoró la situación: en 2009 había 71 asesinados por cada 100 mil y dejó el gobierno del país con 61,1 homicidios en 2014. Sin embargo, con el argumento de que cualquier pacto con la delincuencia era ilegal,

5 "La historia de dimes y diretes iniciaron en 2009 cuando un grupo de diputados areneros inconformes con la presidencia de Alfredo Cristiani abandonaron las filas del partido opositor -la herida por la derrota ante el FMLN y Funes estaba fresca- y formaron Gran Alianza por la Unidad (GANA); desde que nació a la vida legislativa ARENA los acusó una y mil veces de ser corruptos, aunque el principal blanco de las críticas fue el expresidente Saca (Pérez, 2014, p. 2).

6 Como ejemplo, el expresidente Francisco Flores, de ARENA, había estado envuelto en un gran escándalo al haber desaparecido 15 millones de dólares de cooperación procedentes de Taiwán.

7 El FMLN como oposición había criticado fuertemente el endeudamiento público del gobierno por arriba del $40 \%$, pero Funes subió el endeudamiento gubernamental al 59,4 \% al término de su mandato, y en el 2016 Sánchez Cerén ya lo tenía en el 61,4\%. Durante los gobiernos de ARENA hubo un promedio de crecimiento de la economía de 2,56 \% de 2000 a 2008, mientras que el promedio entre 2010 y 2015 fue de solamente $1,98 \%$.
Elecciones presidenciales en EI Salvador 2019: la derrota del FMLN y un nuevo gobierno con Nayib Bukele

Ignacio Medina Núñez 
Sánchez Cerén invalidó cualquier acuerdo y sacó a los militares a las calles, convirtiéndolos incluso en custodios en los centros penales. Así, el segundo gobierno del FMLN provocó la militarización de la seguridad pública, pero con muchos más enfrentamientos en las calles de tal manera que en año y medio de gobierno la cifra de homicidios se elevó en 2015 a 103,6 por cada cien mil, convirtiendo a El Salvador en el país más peligroso de América Latina, superando a Honduras, que había tenido el primer lugar. La popularidad del presidente empezó a decrecer.

$\mathrm{Al}$ ir terminando su cuarto año en el ejecutivo, la percepción sobre el accionar de su gobierno empeoró. No cabe duda de que los gobiernos siempre insistirán en lo que han hecho bien y por ello tal vez Sánchez Cerén en su cuarto informe de gobierno el 1 de junio del 2018 insistía en el éxito de la reforma al sistema de salud, en donde la mortalidad materna era la más baja de la región con solamente 32 muertes; además, de cada mil nacidos vivos en el 2018 solamente había 10 decesos $^{8}$. Se enfatizó mucho en el avance cualitativo del sector educativo, pero ello no se mostró a nivel cuantitativo porque el 2014 comenzó con 3,5 \% ${ }^{9}$ del presupuesto destinado a la educación; en 2015 hubo un ligerísimo incremento al 3,54 \%, pero en el 2016 se bajó al 3,47 \%. También el presidente enfatizaba un número menor de homicidios, comparando con el año 2016, y una reducción mínima de la pobreza. Sin embargo, los resultados de las elecciones para legisladores y munícipes en marzo de 2018 fueron la verdadera prueba donde se mostraba el repudio de la población a su manera de gobernar.

\section{Elecciones legislativas y municipales en 2018}

Las elecciones para legisladores y presidentes de municipios, un año antes de las presidenciales, anunciaron la debacle del FMLN y el repunte de ARENA, al ser éste el principal partido de oposición. Los resultados, aunque sólo participó un 45,8 \% de los votantes, fueron un voto de castigo para el partido en el gobierno: el FMLN vio reducido su número de diputados a 18 (más 5 en alianza con otras fuerzas) mientras que ARENA los elevó a 35 (y 3 más en alianza con el PCN) de un total de 84 en la cámara, en un contexto donde aparecieron más de 200 mil votos nulos, con insultos a la clase política en general. GANA y el PCN quedaban en $3^{\mathrm{a}}$ y $4^{\mathrm{a}}$ posición mientras que PDC y PCN casi desaparecían con siglas propias. "En esa elección el FMLN obtuvo 437 mil votos, es decir, 400 mil votos menos que en la elección legislativa de 2015

\footnotetext{
8 Estos datos fueron proporcionados por Tele Sur el 1 de junio de 2018: "Logros en los 4 años de gestión de Sánchez Cerén en El Salvador”. https://www.telesurtv.net/news/sanchezceren-gestion-20180531-0032.html

9 El mismo Mauricio Funes, quien había dedicado en 2013 un presupuesto de 3,56 a la educación, lo bajó a 3,51 \% en el 2014 y a 3,54 \% en el 2015. Todos estos datos mencionados tienen la fuente en el estudio de S. Arauz, F. Peña y A. Burgos (2016), con base en fuentes oficiales.
} 
y 1,1 millones de votos menos que en la elección presidencial de 2014" (Cartagena, 2019). Este nuevo contexto político en la asamblea legislativa (20182021) se traslada ahora al nuevo gobierno de Bukele (2019-2024) porque, como veremos, su partido GANA solamente puede apoyar al ejecutivo en la presidencia con 10 diputados de los 84 totales.

\begin{tabular}{lcc}
\hline PARTIDOS & VOTOS POR PARTIDO & DIPUTADOS \\
\hline ARENA & 854.651 & 35 \\
\hline FMLN & 437.759 & 18 \\
\hline GANA & 243.267 & 10 \\
\hline PCN & 221.801 & 8 \\
\hline ARENA-PCN & 74.717 & 3 \\
\hline FMLN-CD & 65.027 & 3 \\
\hline PDC & 60.016 & 2 \\
\hline FMLN-PSD-CD & 33.936 & 1 \\
\hline FMLN-PSD & 32.640 & 1 \\
\hline PDC-PCN & 31.315 & 1 \\
\hline FPS & 20.026 & 1 \\
\hline CD & 19.590 & 0 \\
\hline PSD & 15.231 & 1 \\
\hline NO PARTIDARIOS & 14.545 & TOTAL DIPUTADOS: 84 \\
\hline TOTAL DE VOTOS: & $\mathbf{2 . 1 2 4 . 5 2 8}$ & \\
\hline
\end{tabular}

Fuente: Escrutinio final del Tribunal Supremo Electoral (TSE, 2018). https://www.tse.gob. sv/documentos/elecciones/2018/escrutinio-final/reporte/diputaciones-nivel-nacionalelecciones-2018.pdf

En otro ámbito, el gobierno del FMLN mantuvo alianza con el gobierno de Nicolás Maduro en Venezuela, con Daniel Ortega en Nicaragua y con Evo Morales en Bolivia y, por ello, fue duramente criticado por el presidente Donald Trump, un mandatario que aborrece a todos los gobiernos progresistas e izquierdistas de la región latinoamericana. De hecho, el gobierno de Trump, para manifestar su aversión al FMLN, había declarado el término de un acuerdo firmado en 2001 sobre un estatus de protección temporal (TPS) para cerca de 190 mil salvadoreños radicados en Estados Unidos, los cuales, en 2019 tendrían que salir del país o permanecer en territorio norteamericano de forma ilegal. La amenaza del posible retorno de salvadoreños al país era una seria amenaza para la economía de El Salvador tanto por la pérdida de remesas como por el aumento del desempleo al interior del país. En este sentido, el gobierno norteamericano se constituyó también en actor importante contra el FMLN con una propaganda constante en los medios de comunicación para
Elecciones presidenciales en EI Salvador 2019: la derrota del FMLN y un nuevo gobierno con Nayib Bukele Ignacio Medina Núñez

Tabla 1.

Diputados para la asamblea legislativa: elecciones 2018 
Tabla 2.

Alcaldías por partido político o coalición: elecciones 2018

\begin{tabular}{|l|c|}
\hline ARENA & 138 \\
\hline FMLN & 61 \\
\hline GANA & 25 \\
\hline PCN & 24 \\
\hline PDC & 2 \\
\hline PSD & 1 \\
\hline FPS & 1 \\
\hline ARENA-PCN & 2 \\
\hline FMLN-CD & 1 \\
\hline FMLN-CD-PSD-PSP & 1 \\
\hline FMLN-GANA & 1 \\
\hline GANA-FMLN-CD & 1 \\
\hline PCN-PDC & 1 \\
\hline PDC-FMLN & 1 \\
\hline PDC-PCN & 2 \\
\hline TOTAL DE ALCALDÍAS: & 262 \\
\hline
\end{tabular}

Fuente: Escrutinio final del Tribunal Supremo Electoral (TSE, 2018). https://www.tse. gob.sv/documentos/elecciones/2018/escrutinio-final/reporte/alcaldias-nivel-nacionalelecciones-2018.pdf

influir en su contra en el proceso electoral: "Trump amenaza con cortar toda la ayuda estadounidense al país centroamericano, porque según su opinión, el gobierno no logra contener la migración, ni el paso de inmigrantes por su territorio, a pesar de que recortar la ayuda estadounidense de golpe podría agravar aún más los problemas que provocan la migración de salvadoreños"; "La administración Trump ha mostrado una actitud agresiva hacia El Salvador desde el inicio de su gobierno"; "La expansión de noticias falsas ha contribuido a opacar a las verdaderas causas y los responsables de casos de corrupción en el país". Incluso, Trump llegó a nombrar a El Salvador como un shithole country ${ }^{10}$ (Programa de las Américas, 30 enero 2019).

Pero el contexto de violencia generalizada era el argumento interno más importante para la población en su desaprobación al gobierno. El número de homicidios por cada cien mil habitantes podía tener variaciones cada año,

10 Esta expresión "países de mierda” entró al léxico del presidente D. Trump refiriéndose a migrantes: "On January 11, 2018, during an Oval Office talk with several U.S. senators about protecting immigrants from Haiti, El Salvador, and African countries in a new immigration package, President Donald Trump unleashed a word that Americans aren't accustomed to hearing from their president. "Why are we having all these people from shithole countries come here?" The Atlantic: https://www.theatlantic.com/politics/archive/2019/01/shithole-countries/580054/ 
pero los problemas se agravaban: la situación de violencia generalizada junto con la falta de esperanza en los empleos o de un mejoramiento de la economía y en un contexto donde las pandillas ejercían su ley a pesar de la militarización de la seguridad pública.

En todo análisis cuentan los hechos, pero también la percepción de los hechos. Por eso, en un artículo del New York Times se señalaba lo siguiente: "Transcurridos dos gobiernos del FMLN, uno de cada tres hogares salvadoreños aún vive bajo el umbral de pobreza; el salario que recibe un campesino que se desloma en un cafetal es de 200 dólares al mes; tres de cada cinco personas creen que con la actual gestión la atención en los hospitales ha empeorado; y hemos pasado de 3.179 homicidios en 2008 a 3.954 en 2017, un aumento del $24 \% " 11$.

Además, con base en las encuestas de IUDOP que hemos consultado y que fueron realizadas con gran seriedad ${ }^{12}$, se pueden hacer las siguientes consideraciones sobre el gobierno de Sánchez Cerén al finalizar el cuarto año de su gobierno. La preferencia sobre los partidos era la siguiente: ARENA conservaba el primer lugar con una preferencia del 26,7 \%, el FMLN había descendido al $15 \%$, el partido Nuevas Ideas que estaba en formación, todavía sin aprobarse, tenía el 4,7\%, y GANA solamente el 1,7\%. Lo notable era que quienes declaraban no tener ningún partido eran el 48.8 \% (IUDOP, 2018, p. 13). El problema principal del país seguía siendo la delincuencia e inseguridad y así lo señalaba el 52,3\% de los encuestados.

De esta manera, al fijarse en el gobierno de Sánchez Cerén, el 71,1 \% señalaba que con él no había habido ningún cambio positivo en el país: el 56,7 \% no le reconocía ningún logro, y sólo el 22,4 \% alcanzaba a ver en su gobierno como algo bueno la entrega de paquetes escolares. A la pregunta expresa sobre la existencia de cambios negativos en el país con Sánchez Cerén como presidente, el 68,9 \% lo afirmaba con claridad, señalando $31,7 \%$ que su principal fracaso fue el aumento de la delincuencia, entendiendo el 70,9 \% que este fenómeno había crecido en su administración. La evaluación general de su gestión como presidente, el $36 \%$ la consideraba mala, más el 19,5\% que la consideraba muy mala. El 56,3\% pensaba que no había recibido ningún beneficio de su gobierno, y el 57,5\% asumía que El Salvador estaba peor con el gobierno de Sánchez Cerén y el 69,3 \% creía que la situación económica había empeorado. La calificación final sobre su desempeño en el gobierno fue de 4,6 en una escala de 0 a 10.

${ }^{11}$ Artículo de Roberto Valencia en el New York Times, el 30 de enero 2018: "Adiós al bipartidismo en El Salvador", https://www.nytimes.com/es/2018/01/30/opinion-valencia-el-salvadorbipartidismo/

${ }^{12}$ Esta encuesta de 2018 del IUDOP tuvo, igual que las anteriores, una metodología seria y muy detallada y tenía "el objetivo de conocer las opiniones de los salvadoreños sobre el trabajo de la administración Sánchez Cerén en su cuarto año de Gobierno, se efectuó entre el 21 de abril y el 1 de mayo del año en curso, con una muestra nacional de 1.263 entrevistas a personas adultas, la cual es representativa de toda la población de 18 años y más que vive en el país. El estudio cuenta con un error muestral de más o menos 2,8\%" (IUDOP, 2018b, p. 1).
Elecciones presidenciales en EI Salvador 2019: la derrota del FMLN y un nuevo gobierno con Nayib Bukele Ignacio Medina Núñez 


\section{Elecciones presidenciales en 2019}

Durante los últimos años de gobierno del FMLN, empezó a resaltar la figura del político y empresario Nayib Bukele, alumno de los jesuitas en la Universidad Centroamericana José Simeón Cañas, quien había llegado a la alcaldía de Nuevo Cuscatlán en 2012 con el 50,60 \% de votación bajo las siglas del FMLN en alianza con Cambio Democrático (CD), desplazando a ARENA del municipio bajo el gobierno de Funes. Su imagen personal y su trabajo como alcalde le dieron la oportunidad, también cobijado por el FMLN, de llegar a la alcaldía más importante del país, San Salvador, en el 2015, en alianza con el Partido Salvadoreño Progresista (PSP) con el 50,37 \% de votos, desplazando también a ARENA, que ya llevaba dos períodos consecutivos en la capital. Sin embargo, a pesar de su gran carisma y las obras que empezó a realizar en San Salvador, fue expulsado sorpresivamente del FMLN el 10 de octubre de 2017. Sobre este hecho se aducen dos causas: se le acusaba de que no quería seguir los lineamientos emanados por el partido; se le acusó de haber proferido insultos a una regidora del FMLN que le reclamaba más fidelidad al partido. El hecho es que el Tribunal de Ética del FMLN lo expulsó de la organización, aunque él siguió gobernando de manera independiente y manteniendo buena imagen ante la población con acciones en beneficio de la municipalidad.

En 2018, Bukele dejó la alcaldía de San Salvador (la cual fue ganada por ARENA) pero aspiraba a ser candidato presidencial en 2019, ya fuera del FMLN, con un partido propio. Formó un movimiento con el nombre de Nuevas Ideas en octubre de 2017, y llegó a recolectar unas 200 mil firmas (se necesitaban sólo unas $50 \mathrm{mil}$ y se le reconocieron unas $175 \mathrm{mil}$ ) con el objeto de poder convertirlo en un partido político, las cuales fueron presentadas en mayo de 2018; pero el TSE realizó un procedimiento demasiado lento cuando se acercaba la fecha límite para presentar a los candidatos presidenciales de 2019. Por ello, Bukele, en junio 2018, anunció una alianza de su movimiento Nuevas Ideas con un partido legalmente reconocido, Cambio Democrático (CD), de centro izquierda, con el fin de presentarse como candidato para las elecciones presidenciales del siguiente año. Sin embargo, la Sala de lo Constitucional en El Salvador hizo caso a una demanda para desconocer dos partidos (CD y Partido Social Demócrata PSD) por el hecho de no haber alcanzado los votos mínimos necesarios en las elecciones de 2015. El 26 de julio de 2018, el TSE canceló el partido CD. Era manifiesto que desde las esferas gubernamentales se quería impedir la postulación de Bukele a las presidenciales. De esta manera, debido a la demora en el reconocimiento de su movimiento Nuevas Ideas como partido ${ }^{13}$, y también debido a que le cerraron la posibilidad de alianza con CD, Bukele buscó una alianza con GANA,

13 El TSE finalmente accedió a reconocer legalmente Nuevas Ideas como partido el 21 de agosto del 2018, pero en aquella fecha ya había cerrado el registro de candidatos para participar en el proceso electoral presidencial de 2019. 
partido de derecha que había surgido en 2010 como escisión de ARENA. Así, el exalcalde de San Salvador, el mismo 26 de julio de 2018 -posterior al anuncio de cancelación del registro de CD- manifestó públicamente su afiliación a GANA con la intención de ser candidato presidencial, compitiendo en las elecciones internas de ese partido para convertirse en el candidato oficial. Las dos partes (Bukele y GANA) vieron una conveniencia mutua en una candidatura que resultaría finalmente ganadora en 2019; incluso GANA cambió de colores y símbolos para tratar de ser semejante a lo presentado por la organización Nuevas Ideas.

El escenario de los principales partidos contendientes quedó definido: todas las encuestas auguraban la debacle del FMLN por el rechazo a la figura del presidente Sánchez Cerén ${ }^{14}$; el ascenso de la figura de Bukele con GANA era evidente minando las perspectivas descendentes de ARENA que había confiado en regresar al gobierno a partir de los resultados favorables de las legislativas de 2018.

Los candidatos a la presidencia para 2019 fueron los siguientes:

- GANA (Gran Alianza por la Unidad Nacional): Nayib Bukele

- ARENA en alianza con PCN, DS y PDC (Alianza por un Nuevo País): Carlos Calleja

- FMLN: Hugo Martínez

- VAMOS: José Alvarado

El padrón electoral tenía 5.268.411 mientras que en el exterior del país solamente había 5.948 empadronados. Después de la elección del 3 de febrero de 2019 se comprobó que solamente hubo 2.617 .562 votos válidos.

\begin{tabular}{|c|c|c|}
\hline PARTIDO O COALICIÓN & CANDIDATO & VOTOS \\
\hline GANA & Nayib Bukele & 1.434 .856 \\
\hline ARENA-PCN-PDC-DS & Carlos Calleja & 857.084 \\
\hline FMLN & Hugo Martínez & 389.289 \\
\hline VAMOS & José Alvarado & 20.763 \\
\hline Sólo ARENA: & & \\
\hline Sólo PCN: & & \\
\hline Sólo PDC: & & \\
\hline Sólo DS: & & \\
\hline
\end{tabular}

Fuente: Escrutinio final del Tribunal Supremo Electoral (TSE, 2019). https://www.tse.gob. sv/2019/escrutinio-final/presidencial/index.html

14 Todavía después del proceso electoral 2019, la Prensa Gráfica ofreció los resultados de una encuesta el 5 de marzo de 2019, en donde el presidente Salvador Sánchez Cerén era reprobado por el $52 \%$ de los salvadoreños. https://www.laprensagrafica.com/presidente_salvador_sxnchez_cerxn_fue_reprobado_por_el_52x_de_los_salvadorexos-vf20190305mp4.html\#cxrecs_s
Elecciones presidenciales en EI Salvador 2019: la derrota del FMLN y un nuevo gobierno con Nayib Bukele Ignacio Medina Núñez

Tabla 3.

Resultados de las elecciones presidenciales 2019 
El resultado fue la victoria de GANA con el 53,10 \% de votos en la primera vuelta electoral, con lo cual no habría segunda ronda electoral. La figura de Bukele fue determinante para este triunfo. Por otro lado, ARENA superó ampliamente al FMLN, pero quedó muy atrás del fenómeno Bukele. Lo más notable, aunque ya previsible por los resultados de las legislativas de 2018 fue el descenso del FMLN a una $3^{a}$ posición muy distante detrás de GANA y de ARENA. Otros partidos dejaron de tener importancia política por su mínima votación.

La noche del 3 de febrero de 2019 será recordada no solo como aquella en la que el FMLN perdió el gobierno sino también como la que rompió la hegemonía electoral mantenida por los dos partidos mayoritarios desde la firma de los Acuerdos de Paz de 1992. No hay duda de que ambos partidos fueron derrotados a varios niveles; el FMLN, por ejemplo, perdió dos terceras partes de los votantes que le dieron una segunda oportunidad en 2014, al ganar el Ejecutivo. Por otra parte, dejaron a este partido en niveles de votación solo vistos en los años 1997 y 1999, es decir, más de 20 años atrás. Arena, por su parte, acumuló la tercera derrota al hilo, lo que reduce aún más su esperanza de volver a gobernar. (Cartagena, 2019)

La encuesta de La Prensa Gráfica a 1.520 personas mostrada en la televisión el 5 de marzo de 2019, posterior al proceso electoral, reafirmaba las tendencias previas: el 51,9 \% desaprobaba el gobierno de Sánchez Cerén, sobre todo a través de sus grandes fracasos en la lucha contra la inseguridad y el desempleo; el 57 \% de los salvadoreños creía que no había cumplido con todas sus promesas de campaña. Los dos gobiernos del FMLN no pudieron distinguirse de la manera de gobernar de los de ARENA. Esta situación política combinada con el surgimiento de la figura sorprendente y esperanzadora de Bukele fueron la clave explicativa de los resultados de las elecciones presidenciales de 2019.

\section{Conclusiones}

El nuevo gobierno de El Salvador electo en febrero de 2019 en una primera vuelta tomó posesión el 1 de junio del mismo año. De manera semejante a la esperanza que en su momento despertó el FMLN en 2009, Bukele está experimentando su período de 5 años hasta 2024 para mostrar su nuevo proyecto frente a lo hecho por los anteriores partidos políticos.

A partir de lo expuesto en los apartados anteriores, las conclusiones son las siguientes:

1. El proyecto de una izquierda progresista con un nuevo programa de gobierno diferente al modelo neoliberal de los períodos de ARENA como organización de ultraderecha no se pudo mostrar en diez años de gobierno del FMLN. Se puede comprobar que hay una gran diferencia entre ser 
oposición y ser grupo gobernante. Tener las cualidades de una fuerte oposición no siempre cualifica, al convertirse en partido ganador, para ser un buen gobernante. Y esto es muy importante porque hay que entender las enormes dificultades de gobernar con una oposición legislativa fuerte en una división de poderes y con un poder judicial que viene heredado de gobiernos anteriores. La toma de poder del gobierno por un partido opositor no necesariamente significa la toma de todos los poderes del Estado y, por tanto, la mayor cualidad que se necesita es la capacidad de formar consensos en torno a nuevas iniciativas de ley y a la promoción de proyectos específicos. El FMLN, al pasar de la oposición a ser gobierno, tuvo que moderar su actuar político, pero no fue consecuente con sus grandes promesas de cambio social.

2. Los dos gobiernos del FMLN no lograron sacar adelante dos importantes demandas de la población y de numerosos sectores sociales: por un lado, un combate más efectivo a la delincuencia y la corrupción y, por otro, una mejora significativa en la economía nacional en beneficio de amplios sectores de la población. La mala percepción sobre los políticos en el sentido de que muchos partidos son solamente más de los mismos se ha profundizado en El Salvador y, por ello, ha ocurrido la decepción no solamente con relación al partido gobernante, sino también en relación con lo político en general cuando hay tantas papeletas en blanco y cuando no concurre más que la mitad de los electores registrados para votar en momentos tan importantes.

3. Junto con la ineficiencia en el actuar como gobierno para un nuevo proyecto de país, el FMLN no pudo recapacitar para corregir sus divisiones internas. En el proceso histórico, ahora se puede ver que fue un gran error la expulsión de Nayib Bukele en el 2017 cuando era alcalde de San Salvador. $\mathrm{Si}$ a los compañeros de partido es tan fácil expulsarlos y convertirlos en enemigos políticos, resulta muy difícil consolidar una organización fuerte con capacidad de promover los cambios fundamentales para la nación.

4. En la democracia electoral que vivimos, los partidos que en su momento son derrotados ante las nuevas opciones que surgen tienen varias posibilidades: sumergirse en un proceso de debacle descendente que casi los lleve a la extinción o convertirse de nuevo en opción frente a los ciudadanos con la decepción sobre el actuar de los nuevos gobernantes. ARENA gobernó 20 años desde 1989 hasta 2009 en El Salvador y mostró sus grandes deficiencias en los diferentes gobiernos al no hacer crecer la economía ni provocar una mínima redistribución de los recursos sociales junto con terribles actos de corrupción y mala administración, sin embargo, con los dos siguientes gobiernos del FMLN también con malos resultados, ARENA tuvo la oportunidad de regresar con cierto éxito a la arena política a pesar de sus fracasos anteriores, y por eso vimos su recuperación político-electoral en la asamblea legislativa y municipios electos en el 2018 (35 diputados con otros más de sus aliados de un total de 84 , y 138 presidencias
Elecciones presidenciales en EI Salvador 2019: la derrota del FMLN y un nuevo gobierno con Nayib Bukele

Ignacio Medina Núñez 
municipales de 262 totales) que les hicieron tener esperanzas de recuperar el gobierno para el siguiente año. El FMLN comenzó a vivir en 2019 un momento de profunda crisis por las derrotas legislativas y presidenciales, sin embargo, tiene también en adelante la posibilidad de renovarse en su dirección con nuevas estrategias para otras competencias políticas en el futuro.

5. El nuevo gobierno de Bukele en El Salvador ofrece más preguntas que certezas, aunque ciertamente ha provocado grandes esperanzas en la población salvadoreña. Decía Malcolm Cartagena después de estas elecciones:

El nuevo presidente de El Salvador es una incógnita para todos. Nayib Bukele, de 37 años y exmiembro del Frente Farabundo Martí de Liberación Nacional, triunfó por el hartazgo de la ciudadanía frente a los partidos tradicionales. Sin embargo, su figura tampoco está exenta de críticas. Este exalcalde de San Salvador y exitoso empresario mezcla ideas progresistas con valores del emprendedurismo capitalista. (Cartagena, 2019)

La gran pregunta es sobre el tipo de proyecto de país que tiene el gobierno de Bukele en alianza con GANA con un difícil entorno legislativo donde su partido solamente tiene 10 diputados, ARENA tiene 35 y el FMLN 18. Habrá que ver sus primeros resultados de gobierno en la percepción de la población cuando ocurran las elecciones legislativas de 2021.

Ciertamente, desde finales del siglo XX, con la implementación de procesos electorales en América Latina se ha dado un gran paso para intentar terminar con las dictaduras y golpes de Estado, aunque estos últimos todavía persisten como lo sucedido en Brasil 2002, en Honduras 2009 y Bolivia 2019, e incluso con los golpes de Estado legislativos en Paraguay 2012 y en Brasil 2016. Estar realizando elecciones efectivas y con resultados transparentes ha sido un gran avance hacia la democracia, aunque esta tiene proyecciones de participación ciudadana que van mucho más allá de las elecciones. Sin embargo, en momentos determinados, las elecciones resultan muy importantes para consolidar o cambiar el rumbo económico y político de un país, sea hacia proyectos conservadores o progresistas. Para ello, hemos tratado de mostrar la coyuntura específica de las elecciones presidenciales de 2019 en El Salvador, donde un gobierno progresista, habiendo estado 10 años en el poder ejecutivo, fue desechado -junto con el otro partido tradicional de ultraderecha (ARENA) - por el voto ciudadano para dar lugar a un nuevo proyecto de país, cuyos resultados tendrán que ser evaluados de nuevo por los salvadoreños en las elecciones legislativas del 2021. 


\section{Referencias bibliográficas}

Arauz, S., Peña, F. y Burgos, A. (2016). El Salvador. Los cambios que no llegaron. Elfaro. net, 1 de junio 2016. Recuperado de http://elfaro.net/es/201606/el_salvador/18667/Loscambio-que-no-llegaron.htm

Arnson, C. (1994). El Salvador Darkening Horizons: Human Rights on the Eve of the March 1994 Elections. Human Rights Watch /Americas, Report. March 1994, vol. VI, no. 4. Recuperado de http://www.hrw.org/reports/pdfs/e/elsalvdr/elsalv943.pdf

González Díaz, M. (1 de febrero 2019). Elecciones en El Salvador. BBC News Mundo (2019).

Cartagena, M. (18 de febrero de 2019). Nayib Bukele: un presidente "millenial" para El Salvador. Nueva Sociedad. Recuperado de www.nuso.org

CNN Español (3 de febrero de 2019). Nayib Bukele gana las elecciones en El Salvador. Recuperado de https://cnnespanol.cnn.com/2019/02/03/elecciones-el-salvador-salvadorenoseligen-presidente-nuevo/

ERCA Estado de la Región (2016). Quinto Informe Estado de la Región en Desarrollo Humano Sostenible 2016. Un informe desde Centroamérica y para Centroamérica. Costa Rica. Recuperado de www.estadonacion.or.cr

FMLN (2009). Cambio en El Salvador para vivir mejor: Programa de Gobierno 2009-2014. San Salvador, El Salvador.

Funes, M. (2009). Discurso de Mauricio Funes, presidente electo de El Salvador, 1 junio 2009. Recuperado de http://alainet.org/active/29443\%E3\%80\%88=es

IUDOP (2010). Encuesta de evaluación del primer año de gobierno de Mauricio Funes, Asamblea Legislativa y Alcaldías. Consulta de opinión pública mayo 2010. Instituto Universitario de Opinión Pública. San Salvador: Universidad Centroamericana Simeón Cañas.

IUDOP (2013). Encuesta de evaluación del cuarto año de gobierno de Mauricio Funes, Asamblea Legislativa y Alcaldías. Consulta de opinión pública Mayor 2013. Instituto Universitario de Opinión Pública. San Salvador: Universidad Centroamericana Simeón Cañas.

IUDOP (2014a). Encuesta de evaluación del cuarto año de gobierno de Mauricio Funes, Asamblea Legislativa y Alcaldías. Consulta de opinión pública mayo 2014. Instituto Universitario de Opinión Pública. San Salvador: Universidad Centroamericana Simeón Cañas.

IUDOP (2014b). Evaluación del gobierno de Mauricio Funes y del pasado proceso electoral. Instituto Universitario de Opinión Pública. San Salvador: Universidad Centroamericana Simeón Cañas. Recuperado de http://www.uca.edu.sv/publica/iudop/ archivos/presentacion4_2014.pdf

IUDOP (2018). Encuesta de Evaluación del $4^{\circ}$ año de gobierno de Salvador Sánchez Cerén, Asamblea Legislativa, Corte Suprema de Justicia y evaluación postelectoral. Consulta de Opinión Pública de abril-mayo 2018. Instituto Universitario de Opinión Pública. San Salvador: Universidad Centroamericana Simeón Cañas.

IUDOP (2018b). Boletín de Prensa. Año XXXII. No. 3. Instituto Universitario de Opinión Pública. San Salvador: Universidad Centroamericana José Simeón Cañas. Recuperado de http://www.uca.edu.sv/iudop/
Elecciones presidenciales en El Salvador 2019: la derrota del FMLN y un nuevo gobierno con Nayib Bukele

Ignacio Medina Núñez 
Laínez L. y Flores R. (4 de febrero de 2019). Nayib Bukele se declara ganador de las elecciones. La Prensa Gráfica. Recuperado de https://www.laprensagrafica.com/elsalvador/ Nayib-Bukele-se-declara-ganador-de-las-elecciones-20190203-0384.html\#cxrecs_s

La Prensa Gráfica (5 de marzo de 2019). Presidente Salvador Sánchez Cerén fue reprobado por el $52 \%$ de los salvadoreños. San Salvador. Recuperado de https://www.laprensagrafica. com/presidente_salvador_sxnchez_cerxn_fue_reprobado_por_el_52x_de_los_ salvadorexos-vf20190305mp4.html\#cxrecs_s

La Universidad (2009). Cien días de gobierno de Mauricio Funes. La Universidad: órgano científico-sociocultural de la Universidad de El Salvador. Nueva Época, no. 8, octubrenoviembre.

Malamud, C. y Núñez, R. (31 de enero de 2019). Elecciones en El Salvador (2019): un fiel reflejo del momento político latinoamericano. Real Instituto Elcano. Recuperado de http://www.realinstitutoelcano.org/wps/portal/rielcano_es/contenido?WCM_GLOBAL_ CONTEXT=/elcano/elcano_es/zonas_es/ari13-2019-malamud-nunez-elecciones-ēsalvador-fiel-reflejo-momento-politico-latinoamerica

Medina Núñez, I. (comp.) (1995). El Salvador: elecciones y proceso de paz. México: Ediciones de la Universidad de Guadalajara.

Medina Núñez, I. (2005). Democracia y participación ciudadana: la herencia de la cultura política griega. En R. Salazar y P. Lenguita (coords.). Democracia Emancipatoria. Buenos Aires: Editorial Libros en Red.

Medina Núñez, I. (2009). Elecciones presidenciales en América Latina: el surgimiento de una izquierda heterogénea. Buenos Aires: Ediciones elaleph.com.

Medina Núñez, I. (coord.) (2018). Democracia sub-alterna y Estado hegemónico. Crítica política desde América Latina. Diálogo abierto con Álvaro B. Márquez-Fernández. Buenos Aires: Editorial El Pregonero, El Colegio de Jalisco, Elalpeh.

Morlino, L. (2014). La calidad de las democracias en América Latina. Informe para IDEA Internacional. San José, Costa Rica: Instituto Internacional para la Democracia y la Asistencia Electoral (IDEA Internacional).

ONU (1993). De la locura a la esperanza. Informe elaborado por la Comisión de la Verdad en cumplimiento de los Acuerdos de PAZ para El Salvador. San Salvador, Nueva York: Naciones Unidas.

PDA (Political Database of the Americas) (20 de marzo de 2009). El Salvador: 2009 Presidential Elections. Georgetown University and the Organization of American States. Recuperado de http://pdba.georgetown.edu/Elecdata/ElSal/pres09.html

Pérez, D. E. (6 de febrero de 2014). FMLN incorpora a sus filas el saquismo sin Saca. ALAI (América Latina en Movimiento). Recuperado de http://alainet.org/active/71132

Petrich, B. (2009). Déficit fiscal de más de mil millones de dólares, herencia de Arena a Mauricio Funes. Univision.com. Recuperado el 29 de mayo de $2009 \mathrm{http}$ ///foro.univision. com/univision/board/message?board.id=politicaelsalvador\&message. $.1 d=72500$

Programa de las Américas (30 de enero de 2019). En El Salvador, el combate a la corrupción quedó corto. Program Director: Laura Carlsen. Recuperado de https://www.americas.org/es/ en-el-salvador-el-combate-a-la-corrupcion-quedo-corto/?utm

Spinoza, B. (2015). Tratado teológico-político. Epublibre. 21-02-2015. Traducción de Atilano Domínguez de la versión original de 1670.

Touraine, A. (2001). ¿Qué es la democracia? México: Fondo de Cultura Económica. 
Transparencia Activa (26 de marzo de 2014). Tribunal Supremo Electoral entrega credenciales a presidente y vicepresidente electos. Recuperado de http://www.transparenciaactiva.gob.sv

TSE (7 de febrero de 2019). Tribunal Supremo Electoral de El Salvador. Escrutinio final. Recuperado de https://www.tse.gob.sv/2019/escrutinio-final/presidencial/index.html

Wolf, S. (2011). Peace without justice: Violence, Marginality, and the struggle to build a new El Salvador. Journal of Iberian and Latin American Research, vol. 17, no. 1, July, pp. 115-119. Publicado en línea el 30 de junio de 2011. DOI: https://doi.org/10.1080/1326 0219.2011.579893

Wolf, S. (February 22, 2019). Will El Salvador's New President Live Up to the Image People Voted for?. The Globe Post. Recuperado de https://theglobepost.com/2019/02/19/ el-salvador-new-president/
Elecciones presidenciales en EI Salvador 2019: la derrota del FMLN y un nuevo gobierno con Nayib Bukele Ignacio Medina Núñez 